\title{
Semi-Global Existence and Convergence of Solutions of the Robinson-Trautman (2-Dimensional Calabi) Equation
}

\author{
Piotr T. Chruściel ${ }^{\star} \star \star$ \\ Courant Institute, New York University, 251 Mercer Street, New York, NY 10012, USA
}

Received December 1, 1989; in revised form June 4, 1990

\begin{abstract}
It is shown that for smooth initial data solutions of the RobinsonTrautman equation (also known as the two-dimensional Calabi equation) exist for all positive "times," and asymptotically converge to a constant curvature metric.
\end{abstract}

\section{Introduction}

One of the most interesting problems in classical general relativity is the understanding of the formation - or lack thereof - of singularities in space-time in the course of evolution of the metric via Einstein equations. Since the tools to analyze this problem in its whole generality do not seem to be available yet, it is of interest to try to understand such issues under various restrictive hypotheses, e.g. smallness of initial data (cf. e.g. $[6,11]$ ), or under some symmetry hypotheses $[5,20$, $16,8,7]$, or both, or under some other restrictions (cf. e.g. [9]). In this paper we prove semi-global existence and convergence for a class of vacuum solutions of Einstein equations known as the Robinson-Trautman metrics [25], thus no curvature singularities (other than the singularity $r=0$ which is already present in the initial data set) develop for finite values of the retarded time ${ }^{1} u$ in this class of metrics. The Robinson-Trautman metrics have played an important role in the early understanding of gravitational radiation, providing the first known class of solutions of Einstein vacuum equations which could be interpreted as representing a gravitationally radiating isolated system. These metrics have the amusing

* On leave of absence from the Institute of Mathematics, Polish Academy of Sciences, Warsaw $\star \star$ Supported in part by NSF grant DMS-885773 to the Courant Institute and by the Polish Ministry of Science Research grant RPBP 01.3

${ }_{1}$ More precisely, no curvature singularities develop for finite positive values of $u$ if the total mass is positive, for negative values of $u$ if the total mass is negative. It should be noted that the space-times the existence of which is established here will still be singular in the sense of being geodesically incomplete, cf. [28] and Proposition 2.1. 
property that the four-dimensional space-time metric, which satisfies a second order hyperbolic system of equations, is constructed from a solution of a fourth order parabolic equation for a Riemannian metric on a two dimensional manifold. At the origin of this is the fact that the initial data for the space-time metric are prescribed on a null (rather than spacelike) hypersurface. As has been stressed by Tod [28], the Robinson-Trautman equation turns out to be the two-dimensional version of an equation considered by Calabi [3] in the context of extremal Kähler metrics. Curiously enough, the problem of local existence and uniqueness of solutions of the Robinson-Trautman equation seems to have been first discussed in the physical literature only in 1988, by Schmidt [26]. The linearized equation has been studied by Foster and Newman [10], by Vandyck [29], and recently by Robinson [24]. Numerical results of Perjes [22] and Singleton [27] suggested semi-global existence (i.e. existence for all $u \geqq u_{0}$ ) and asymptotic convergence of solutions to constant curvature metrics on the two-dimensional manifold ${ }^{2} M$. It was noted by Rendall [23] that this is indeed the case for all "small initial data" unless ${ }^{2} M \approx S^{2}$, the two-dimensional sphere; in this last case A. Rendall showed semi-global existence and convergence provided the initial data satisfied a (rather unsatisfactory) parity condition. Let us finally note that the global structure of the Robinson-Trautman space-times has been studied by Schmidt [26] and Tod [28]. In this paper we show that for all compact, orientable two dimensional topologies and all (say, smooth) initial data solutions of the Robinson-Trautman equation exist for all positive ${ }^{2}$ values of the "retarded time" $u$. The key elements of the proof are a) the Calabi - Lukàcs-Perjes-PorterSebastyén (LPPS) $[3,18]$ monotonicity property of the integral of the square of the scalar curvature, and b) an expression for the Bondi mass of the RobinsonTrautman metrics derived by Singleton [27]. We point out that for smooth nonanalytic initial data no backwards solutions of the Robinson-Trautman equation exist, thus the results presented here are necessarily of a semi-global rather than global character. We also show that all solutions converge exponentially fast to constant curvature metrics.

It is of some interest to compare the results and methods of this paper with the analogous existence and convergence problem for the Ricci flow [14, 4, 21]. One would expect the problem here to be more difficult, due to a lack of the maximum principle for higher order parabolic equations, an extensive use of which is made when studying the Ricci flow. It is the author's belief that the proofs here are actually simpler, though certainly less elegant, than in the Ricci flow case.

This paper is organized as follows: in Sect. 2 we briefly discuss some of the properties of the Robinson-Trautman equation. In Sect. 3 an a priori $L_{\infty}$ bound is derived. In Sect. 4 higher order a priori estimates are established, which prove global existence of solutions. In Sect. 5 asymptotic convergence of solutions to constant curvature metrics is proved.

\section{The Robinson-Trautman Equation}

As has been shown by Robinson and Trautman [25], in every space-time which contains a hypersurface-orthogonal, shear-free, null geodesic congruence, there

\footnotetext{
2 Positive if the total mass is positive, negative if the total mass is negative
} 
exists a coordinate system in which the metric takes the form

$$
\begin{aligned}
d s^{2} & =-\Phi d u^{2}-2 d u d r+r^{2} e^{2 \lambda} \stackrel{\circ}{g}_{a b} d x^{a} d x^{b}, \\
\lambda & =\lambda\left(u, x^{a}\right), \quad \stackrel{\circ}{g}_{a b}=\stackrel{\circ}{g}_{a b}\left(x^{a}\right),
\end{aligned}
$$

where $\stackrel{\circ}{a b}_{a b}$ is a fixed metric on a two dimensional manifold ${ }^{2} M$, which we assume to be compact and orientable, with local coordinates $x^{a}, a=1,2$. As has been discussed by Robinson [24], a suitable redefinition of $u$ and $r$ which preserves (2.1) leads to

$$
\forall u \int_{2_{M}} e^{2 \lambda} d \mu_{0}=\int_{2_{M}} d \mu_{0}, \quad d \mu_{0}=\sqrt{\operatorname{det} \stackrel{\circ}{a b}_{a b}} d^{2} x
$$

Whenever (2.2) holds, Einstein equations imply [25]

$$
\Phi=\frac{R}{2}+\frac{r}{12 m} \Delta_{g} R-\frac{2 m}{r}, \quad R \equiv R(g),
$$

where $m$ is a constant, and $R(g)=R_{a b}^{a b}$ is the curvature scalar of the metric

$$
g_{a b}=e^{2 \lambda} \stackrel{\circ}{a b}_{a},
$$

$\Delta_{g}\left(\Delta_{0}\right)$ denotes the Laplacian of the metric $g(\dot{g})$. If the constraint (2.2) is imposed, Einstein equations for a metric of the form (2.1) reduce to the single first order quasilinear parabolic equation

$$
\frac{\partial \lambda}{\partial u}=\frac{1}{24 m} \Delta_{g} R,
$$

which implies the following equation for $g_{a b}$,

$$
\frac{\partial g_{a b}}{\partial u}=\frac{1}{12 m} \Delta_{g} R g_{a b},
$$

an equation considered by Calabi in [3]. If $m>0$ (2.4) is parabolic for $u$-increasing, while for $m<0$ it is parabolic for $u$-decreasing, thus for generic initial data one does not expect to have existence of global solutions of (2.4), i.e. existence of solutions defined for $(u, p) \in(-\infty, \infty) \times{ }^{2} M$. In fact, every solution of (2.4) immediately becomes analytic [17], thus one has

Proposition 2.1. Let $\lambda_{0}$ be a non-analytic smooth function on ${ }^{2} M$, let $\stackrel{\circ}{g}_{a b}$ be an analytic metric on ${ }^{2} M$. There exists no solution of $(2.4)$ defined on $(-\varepsilon, \varepsilon) \times{ }^{2} M$ such that $\lambda(0, p)=\lambda_{0}(p)$.

Proposition 2.1 shows one-sided non-extendability of the metric (2.1) in the vacuum Robinson-Trautman class whenever the metric (2.1) is smooth but nonanalytic at $u=u_{0}$. This is an amusing example of pathologies which may occur when considering the characteristic initial value problem for Einstein equations. In [8] a similar non-existence result was exhibited, when the null surface is a smooth but non-analytic compact Cauchy horizon. It may well be possible that there exist vacuum extensions beyond a smooth non-analytic surface $u=u_{0}$ which are not in the Robinson-Trautman class.

The above discussion establishes generic non-existence of global solutions of (2.4), it is therefore natural to ask whether "semi-global" solutions exist, i.e. solutions defined for all $u \geqq u_{0}$ when $m>0$, or for all $u \leqq u_{0}$ when $m<0$. As 
stated in the Introduction, we shall show that this is indeed the case - the results of the subsequent sections may be summarized as follows:

Theorem 2.1. Let $\left({ }^{2} M, g\right)$ be a smooth, compact, orientable, two dimensional Riemannian manifold. For any ${ }^{3} \lambda_{0} \in C^{6}\left({ }^{2} M\right)$ there exists a function $\lambda \in C^{1}\left([0, \infty) \times{ }^{2} M\right) \cap C^{\infty}\left((0, \infty) \times{ }^{2} M\right), \lambda(u, \cdot) \in C^{4}\left({ }^{2} M\right)$ for $u \geqq 0$, satisfying (2.4), such that $\lambda(0, p)=\lambda_{0}(p)$ (if $\lambda_{0} \in C^{\infty}\left({ }^{2} M\right)$, then $\left.\lambda \in C^{\infty}\left([0, \infty) \times{ }^{2} M\right)\right)$. Moreover the metric $e^{2 \lambda} \mathrm{g}$ converges exponentially fast ${ }^{4}$ to a constant curvature metric on ${ }^{2} M$.

Before closing this section let us recall that $g$ can always be chosen so that

$$
\partial_{a} R_{0}=0, \quad R_{0} \equiv R(\stackrel{\circ}{g}),
$$

with $R_{0}>0$ on $S^{2}, R_{0}=0$ on $T^{2}, R_{0}<0$ in the remaining cases. Changing the orientation of the $u$-axis if necessary, by a rescaling of $u$ we may always achieve

$$
12 m=1
$$

and in the remainder of this paper we shall always assume that this normalization has been chosen. We have the following formulae:

$$
\begin{gathered}
R=R(g)=e^{-2 \lambda}\left(R_{0}-2 \Delta_{0} \lambda\right), \\
\Delta_{g}=e^{-2 \lambda} \Delta_{0} \\
\frac{\partial \lambda}{\partial u}=\frac{1}{2} \Delta_{g} R, \\
\frac{\partial R}{\partial u}=-\Delta_{g} \Delta_{g} R-R \Delta_{g} R, \\
\frac{\partial}{\partial u} d \mu_{g}=\Delta_{g} R d \mu_{g}, \quad d \mu_{g} \equiv \sqrt{\operatorname{det} g_{a b}} d^{2} x,
\end{gathered}
$$

and we also have

$$
\begin{gathered}
\int_{2_{M}} d \mu_{g}=\int_{2_{M}} e^{2 \lambda} d \mu_{0}=\int_{2_{M}} e^{2 \lambda \theta_{0}} d \mu_{0}=A \\
A \equiv \int_{2_{M}} d \mu_{0},\left.\quad \lambda_{0} \equiv \lambda\right|_{u=0}, \\
\langle R\rangle \equiv \frac{1}{A} \int_{2_{M}} R d \mu_{g}=\frac{1}{A} \int_{2_{M}} R_{0} d \mu_{0}=R_{0}, \\
u \geqq\left. u_{0} \Rightarrow \int\left(R-R_{0}\right)^{2} d \mu_{g}\right|_{u} \leqq\left.\int\left(R-R_{0}\right)^{2} d \mu_{g}\right|_{u_{0}}, \\
u \geqq\left. u_{0} \Rightarrow \int e^{3 \lambda} d \mu_{0}\right|_{u} \leqq\left.\int e^{3 \lambda} d \mu_{0}\right|_{u_{0}} .
\end{gathered}
$$

\footnotetext{
3 As mentioned in Sect. 4, the result still holds with $\lambda_{0} \in W_{2,2}\left({ }^{2} M, d \mu_{0}\right)$, in which case $\lambda \in C\left([0, \infty) ; W_{2,2}\left({ }^{2} M, d \mu_{0}\right)\right) \cap C^{\infty}\left((0, \infty) \times{ }^{2} M\right)$

4 The convergence rate of the metric is made precise in Proposition 5.1; the results of that proposition can be sharpened by establishing an asymptotic expansion of $\lambda$ for large $u$ - these results and their implications for the global structure of the Robinson-Trautman space-times will be discussed elsewhere
} 
Equation (2.9) follows from (2.2); (2.10) is the Gauss-Bonnet theorem; (2.11) is the Calabi-LPPS inequality $[3,18] ;(2.12)$ is D. Singleton's version of the Bondi mass-loss formula [27]; for completeness (2.11) and (2.12) are derived in Appendix A.

\section{Uniform $W_{2,2}$ a priori Estimates}

Let $L_{p} \equiv L_{p}\left({ }^{2} M, d \mu_{0}\right)$ be the space of functions the $p^{\text {th }}$ power of which is integrable on ${ }^{2} M$ with respect to the measure $d \mu_{0}$, let $W_{k, p} \equiv W_{k, p}\left({ }^{2} M, d \mu_{0}\right)$ be the Sobolev space of functions the derivatives of which up to order $k$ are in $L_{p}$. In this section we shall derive an a priori $W_{2,2}$ bound for solutions $\lambda(u)$ of (2.4) such that $\lambda_{0} \equiv \lambda(0) \in W_{2,2}$. Since (2.4) is in divergence form the notion of weak solution for $\lambda \in C\left([0, T) ; W_{2,2}\left({ }^{2} M, d \mu_{0}\right)\right)$ can be defined in a standard way. Proposition 3.1 is the key to the global existence proof and is specific to the problem at hand - the rest of the proof, as carried on in the next sections, is rather standard and applies to a quite general class of equations. We shall thus consider the following problem:

$$
\left\{\begin{array}{l}
\frac{\partial \lambda}{\partial u}=\frac{1}{2} \Delta_{g} R, \\
\lambda \in C\left([0, T) ; W_{2,2}\left({ }^{2} M, d \mu_{0}\right)\right), \quad 0<T \leqq \infty, \\
\lambda(u=0)=\lambda_{0} \in W_{2,2}\left({ }^{2} M, d \mu_{0}\right), \\
\int_{2 M} e^{2 \lambda_{0}} d \mu_{0}=\int_{2 M} d \mu_{0} .
\end{array}\right.
$$

If $\lambda_{0}$ is e.g. smooth, then a solution of (3.1) will be smooth both in $u$ and in $p \in{ }^{2} M$, and will thus be a classical solution of (2.4) (cf. also the remarks at the beginning of the next section). Throughout this paper the letter $C$ denotes a generic constant which may vary from line to line.

Proposition 3.1. Let $\lambda$ satisfy (3.1). There exists a constant $C$ depending only upon $\|\lambda(0)\|_{W_{2,2}}$ and $\stackrel{\circ}{g} a b$ such that

in particular

$$
\forall u \in[0, T)\|\lambda(u)\|_{W_{2,2}} \leqq C,
$$

$$
\forall u \in[0, T) \sup _{p \in M}|\lambda(u, p)| \leqq C .
$$

Proof. Equation (2.5) inserted into (2.11) gives

Hölder's inequality implies

$$
\begin{gathered}
\int e^{-2 \lambda}\left(\Delta_{0} \lambda-\frac{1}{2} R_{0}\right)^{2} d \mu_{0} \leqq C, \\
C=C\left(\left\|\lambda_{0}\right\|_{W_{2,2}}, g_{a b}\right)
\end{gathered}
$$

$$
\begin{aligned}
\int\left|\Delta_{0} \lambda-\frac{1}{2} R_{0}\right|^{6 / 5} d \mu_{0} & =\int e^{6 \lambda / 5}\left|e^{-\lambda}\left(\Delta_{0} \lambda-\frac{1}{2} R_{0}\right)\right|^{6 / 5} d \mu_{0} \\
& \leqq\left(\int e^{3 \lambda} d \mu_{0}\right)^{2 / 5}\left(\int e^{-2 \lambda}\left(\Delta_{0} \lambda-\frac{1}{2} R_{0}\right)^{2} d \mu_{0}\right)^{3 / 5} \\
& \leqq C
\end{aligned}
$$


and we have used (3.4) and D. Singleton's inequality (2.12). Equation (3.5) leads to

$$
\left\|\Delta_{0} \lambda\right\|_{L_{6 / 5}} \leqq C
$$

Let us recall the following, well known fact, which we prove here for completeness:

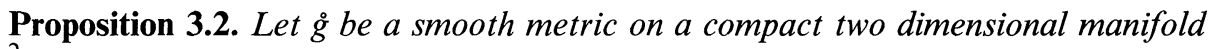
${ }^{2} M$, let $1<p<\infty$, let $\phi \in W_{2, p}$ satisfy

$$
\begin{gathered}
\langle\phi\rangle=\frac{1}{A} \int \phi d \mu_{0}=0, \quad A=\int d \mu_{0}, \\
\Delta_{0} \phi=\rho, \quad \rho \in L_{p}\left({ }^{2} M, d \mu_{0}\right) .
\end{gathered}
$$

There exists a constant $C(\stackrel{\circ}{g})$ such that

$$
\|\phi\|_{W_{2, p}} \leqq C\|\rho\|_{L_{p}} .
$$

Proof. We have the well known estimate

$$
\|\phi\|_{W_{2, p}} \leqq C\left(\|\rho\|_{L p}+\|\phi\|_{L p}\right)
$$

(cf. e.g. [1, Theorem $15.1^{\prime}$, p. 703]; the result presented there extends immediately to manifolds); to establish (3.7) we thus have to show

$$
\|\phi\|_{L p} \leqq C\|\rho\|_{L p} \text {. }
$$

Integrating over $M$ the identity

$$
\dot{\nabla}_{a}\left(\phi \dot{\nabla}^{a} \phi\right)=|\dot{\nabla} \phi|^{2}+\phi \Delta_{0} \phi
$$

one obtains

$$
\begin{aligned}
\int|\nabla \phi|^{2} d \mu_{0} & =-\int \phi \Delta_{0} \phi d \mu_{0} \\
& \leqq\|\phi\|_{L_{q}}\left\|\Delta_{0} \phi\right\|_{L_{p}}, \quad \frac{1}{p}+\frac{1}{q}=1 .
\end{aligned}
$$

From Sobolev's inequality (cf. e.g. [2, Theorem 3.67, p. 91]) and (3.9) one gets

$$
\|\phi\|_{L_{q}} \leqq C\|\nabla \phi\|_{L_{2}} \leqq C\|\phi\|_{L_{q}}^{1 / 2}\|\rho\|_{L_{p}}^{1 / 2} \Rightarrow\|\phi\|_{L_{q}} \leqq C^{2}\|\rho\|_{L_{p}},
$$

and (3.9) yields

$$
\|\dot{\nabla} \phi\|_{L_{2}} \leqq C\|\rho\|_{L_{p}},
$$

(3.8) follows again by Sobolev's inequality.

Returning to the proof of Proposition 3.1, let

$$
\begin{aligned}
\phi & =\lambda-\langle\lambda\rangle, \\
\langle\lambda\rangle & \equiv \frac{1}{A} \int \lambda d \mu_{0}, \quad A=\int d \mu_{0} .
\end{aligned}
$$

Proposition 3.2 and Eq. (3.6) give

$$
\|\phi\|_{W_{2,6 / 5}} \leqq C .
$$


Since (3.11) controls the modulus of continuity of $\lambda$ we are done, an elegant way to proceed is as follows: from Moser's inequality [2, Theorem 2.46, p. 63]

$$
\int e^{2(\lambda-\langle\lambda\rangle)} d \mu_{0} \leqq C \exp \left[\mu\|\phi\|_{W_{1,2}}^{2}\right],
$$

for some constants $C$ and $\mu$, from (2.9) and (3.11) we have

$$
e^{-2\langle\lambda\rangle} \leqq C \Rightarrow\langle\lambda\rangle \geqq-C^{\prime},
$$

on the other hand

$$
\begin{aligned}
& \langle\lambda\rangle=\int_{\Omega^{-}} \lambda d \mu_{0}+\int_{\Omega^{+}} \lambda d \mu_{0} \leqq \int_{\Omega^{+}} \lambda d \mu_{0} \leqq \int_{\Omega^{+}} e^{2 \lambda} d \mu_{0} \leqq C, \\
& \Omega_{-}=\left\{p \in \in^{2} M: \lambda \leqq 0\right\}, \quad \Omega_{+}=\left\{p \in^{2} M: \lambda \geqq 0\right\} .
\end{aligned}
$$

Equations (3.12) and (3.13) thus give

$$
|\langle\lambda\rangle| \leqq C
$$

from (3.10), (3.11), and (3.14) it follows

$$
\|\lambda\|_{W_{2,6 / 5}} \leqq C \text {. }
$$

From (3.15) and the Sobolev embedding [2, Chap. II, Sect. 3, p. 35] we have

$$
\|\lambda\|_{L \infty} \leqq C
$$

(3.16) and (3.4) imply

$$
\int\left(\Delta_{0} \lambda\right)^{2} d \mu_{0} \leqq C ;
$$

(3.14) and Proposition 3.2 establish the claimed assertion.

\section{Higher a priori Estimates}

In this section we derive a priori bounds on all $W_{k, 2}$ norms. This, together with the local existence results discussed e.g. in [26] or [23] shows semi-global existence of solutions ${ }^{5}$. The estimates of Lemma 4.2 can be used to show that $\lambda \in C([0, T)$; $\left.W_{2,2}\left({ }^{2} M\right)\right)$ implies $\lambda \in C^{\infty}\left((0, T) ; C^{\infty}\left({ }^{2} M\right)\right)=C^{\infty}\left((0, T) \times{ }^{2} M\right)$ and thus $\lambda$ is a classical solution for $u>0$ [for $u \geqq 0$ if $\lambda_{0} \in W_{6,2}$ - this will be the case if e.g. $\left.\lambda_{0} \in C_{6}\left({ }^{2} M\right)\right]$.

Proposition 4.1. Let $0<T \leqq \infty, k \geqq 2$, let $\lambda \in C\left([0, T) ; W_{k, 2}\left({ }^{2} M, d \mu_{0}\right)\right)$ be a solution of (3.1), $\lambda(0) \in W_{k .2}\left({ }^{2} M, d \mu_{0}\right)$. There exists a constant $C$ such that

$$
\forall 0 \leqq u<T\|\lambda(u)\|_{w_{k, 2}} \leqq C(1+u)^{1 / 2} .
$$

The proof of Proposition 4.1 makes use of the lemma that follows. Wherever convenient one may suppose that $\lambda \in C^{\infty}\left([0, T) \times{ }^{2} M\right)$, appropriate results under weaker differentiability conditions can then be established by a density argument.

\footnotetext{
5 Let us note that energy estimates similar to the ones derived here can be used to prove local existence of solutions for $\lambda_{0} \in W_{2,2}\left({ }^{2} M\right)$, thus the results of the previous section are actually sufficient to show semi-global existence
} 
In the remainder of this paper we shall assume that $g$ is a smooth metric the Ricci scalar of which is a constant. For any multi-index $\alpha=\left(a_{1}, \ldots, a_{j}\right)$ let

$$
\dot{\nabla}_{\alpha}=\dot{\nabla}_{a_{1}} \ldots \dot{\nabla}_{a_{\jmath}}
$$

where $\dot{\nabla}$ is the covariant derivative of the metric $\stackrel{\circ}{\text {. For }} j \in \mathbb{N}$ set

$$
\left|\dot{\nabla}^{j} \lambda\right|^{2}=g^{a_{1} b_{1}} \ldots \dot{g}^{a_{j} b_{j}} \dot{\nabla}_{a_{1}} \ldots \dot{\nabla}_{a_{j}} \lambda \dot{\nabla}_{b_{1}} \ldots \dot{\nabla}_{b_{j}} \lambda .
$$

Lemma 4.1. For every $l \in \mathbb{N}, l \geqq 1$, there exist a finite set of indices $B$, a set of constants $2 \leqq N(l, \beta)<\infty, \beta \in B$, and a constant $C<\infty$ such that

$$
\begin{gathered}
\frac{d}{d u}\left\|e^{4 \lambda}\left|\dot{\nabla}^{l} \lambda\right|\right\|_{L_{2}}^{2} \leqq-\left\|\dot{\nabla}^{l+2} \lambda\right\|_{L_{2}}^{2}+C \sum_{\beta \in B}\|\phi\|_{W_{2,2}}^{N(l, \beta)}, \\
\phi \equiv \lambda-\langle\lambda\rangle, \quad\langle\lambda\rangle \equiv \frac{1}{A} \int_{2_{M}} \lambda d \mu_{0}, \quad A=\int_{2 M} d \mu_{0} .
\end{gathered}
$$

Moreover there exists a constant $C^{\prime}$ depending also upon $\|\lambda(0)\|_{W_{2,2}}$ such that

$$
\frac{d}{d u}\left\|e^{4 \lambda}\left|\dot{\nabla}^{l} \lambda\right|\right\|_{L_{2}}^{2} \leqq-\left\|\dot{\nabla}^{l+2} \lambda\right\|_{L_{2}}^{2}+C^{\prime}\|\phi\|_{W_{2,2}}^{2} .
$$

Proof. Let

$$
E_{l}=\int_{2 M} e^{4 \lambda}\left|\nabla^{l} \lambda\right|^{2} d \mu_{0}
$$

with $\left|\nabla^{l} \lambda\right|^{2}$ as in (4.2). A straightforward though somewhat lengthy and tedious analysis presented in detail in Appendix C (cf. Lemma C.4) gives

$$
\begin{gathered}
\frac{d E_{l}}{d u} \leqq-2\left\|\nabla^{l+2} \lambda\right\|_{L_{2}}^{2}+C \sum_{\beta \in B}\left\|\dot{\nabla}^{l+2} \lambda\right\|_{L_{2}}^{\alpha_{\beta}}\|\phi\|_{W_{2,2}}^{\gamma_{\beta}}, \\
\alpha_{\beta<2}, \quad \gamma_{\beta} \geqq 2-\alpha_{\beta} .
\end{gathered}
$$

Yang's inequality "with $\varepsilon$,"

$$
a b \leqq \varepsilon a^{p}+\frac{1}{q}(p \varepsilon)^{-q / p} b^{q}, \quad \frac{1}{p}+\frac{1}{q}=1,
$$

with $p=2 / \alpha_{\beta}, q=2 /\left(2-\alpha_{\beta}\right)$, applied to each of the terms appearing in (4.5) gives

$$
\begin{gathered}
\left\|\dot{\nabla}^{l+2} \lambda\right\|_{L_{2}}^{\alpha_{\beta}}\|\phi\|_{W_{2,2}}^{\gamma_{\beta}} \leqq \varepsilon\left\|\dot{\nabla}^{l+2} \lambda\right\|_{L_{2}}^{2}+C(l, \beta, \varepsilon)\|\phi\|_{W_{2,2}}^{N(l, \beta)}, \\
N(l, \beta)=\frac{2 \gamma_{\beta}}{2-\alpha_{\beta}} \geqq 2
\end{gathered}
$$

which proves (4.3) when $\varepsilon$ is chosen small enough. Since

$$
\|\phi(u)\|_{W_{2,2}}^{\gamma_{\beta}}=\|\phi(u)\|_{W_{2,2}}^{\gamma_{\beta}-2}\|\phi(u)\|_{W_{2,2}}^{2} \leqq C\left(\|\phi(0)\|_{W_{2,2}}\right)\|\phi(u)\|_{W_{2,2}}^{2},
$$

for some function $C\left(\|\phi(0)\|_{w_{2,2}}\right)$, (4.3) implies (4.4).

Proof of Proposition 4.1. Integration in $u$ of (4.3) using (3.2) gives

$$
\left\|e^{-4 \lambda} \nabla^{l} \lambda\right\|_{L_{2}}^{2} \leqq C(1+u) \text {, }
$$


and (3.3) implies

$$
\left\|\dot{\nabla}^{l} \lambda\right\|_{L_{2}} \leqq C(1+u)^{1 / 2}
$$

which establishes (4.1).

We shall now derive a "smoothing-out" inequality, which follows from Lemma 4.1. The method of proof follows [15]:

Lemma 4.2. Let $\lambda$ satisfy (3.1), $\lambda\left(u_{0}\right) \in W_{k, 2}, k \geqq 2, u_{0} \geqq 0$. For every $l \geqq 0$ there exists a constant $C_{l, k}$ depending upon $l, k, \stackrel{\circ}{g}$ and $\left\|\lambda_{0}\right\|_{w_{2,2}}$ such that, for $u \geqq u_{0}$,

$$
\begin{aligned}
\left\|\left(\dot{\nabla}^{k+2 l} \lambda\right)(u)\right\|_{L_{2}}^{2} \leqq & \frac{C_{l, k}}{\left(u-u_{0}\right)}\left\{\left\|\left(\dot{\nabla}^{k} \lambda\right)\left(u_{0}\right)\right\|_{L_{2}}^{2}\right. \\
& \left.+\int_{u_{0}}^{u}\left(1+\left(s-u_{0}\right)^{l}\right)\|(\lambda-\langle\lambda\rangle)(s)\|_{W_{2,2}}^{2} d s\right\} .
\end{aligned}
$$

Proof. For $u \geqq u_{0}$ let

$$
\begin{aligned}
E(u)= & \left\|e^{2 \lambda} \dot{\nabla}^{k} \lambda\right\|_{L_{2}}^{2}+c_{1}\left(u-u_{0}\right)\left\|e^{2 \lambda} \dot{\nabla}^{k+2} \lambda\right\|_{L_{2}}^{2} \\
& +\ldots+c_{l}\left(u-u_{0}\right)^{l}\left\|e^{2 \lambda} \dot{\nabla}^{k+2 l} \lambda\right\|_{L_{2}}^{2},
\end{aligned}
$$

where the $c_{i}$ 's are positive constants to be chosen later. By (4.4) we have

$$
\begin{aligned}
\frac{d E}{d u}= & \left(\frac{d}{d u}\left\|e^{2 \lambda} \dot{\nabla}^{k} \lambda\right\|_{L_{2}}^{2}+c_{1}\left\|e^{2 \lambda} \dot{\nabla}^{k+2} \lambda\right\|_{L_{2}}^{2}\right) \\
& +\left(u-u_{0}\right)\left(c_{1} \frac{d}{d u}\left\|e^{2 \lambda} \dot{\nabla}^{k+2} \lambda\right\|_{L_{2}}^{2}+2 c_{2}\left\|e^{2 \lambda} \dot{\nabla}^{k+4} \lambda\right\|_{L_{2}}^{2}\right) \\
& +\cdots+ \\
& +\left(u-u_{0}\right)^{l-1}\left(c_{l-1} \frac{d}{d u}\left\|e^{2 \lambda} \dot{\nabla}^{k+2 l-2} \lambda\right\|_{L_{2}}^{2}+l c_{l}\left\|e^{2 \lambda} \nabla^{k+2 l} \lambda\right\|_{L_{2}}^{2}\right) \\
& +c_{l}\left(u-u_{0}\right)^{l} \frac{d}{d u}\left\|e^{2 \lambda} \dot{\nabla}^{k+2 l} \lambda\right\|_{L_{2}}^{2} \\
\leqq & \left(c_{1} \sup e^{4 \lambda}-1\right)\left\|\dot{\nabla}^{k+2} \lambda\right\|_{L_{2}}^{2} \\
& +\left(u-u_{0}\right)\left(2 c_{2} \sup e^{4 \lambda}-c_{1}\right)\left\|\dot{\nabla}^{k+4} \lambda\right\|_{L_{2}}^{2} \\
& +\cdots+ \\
& +\left(u-u_{0}\right)^{l-1}\left(l c_{l} \sup e^{4 \lambda}-c_{l-1}\right)\left\|\dot{\nabla}^{k+2 l} \lambda\right\|_{L_{2}}^{2} \\
& +\sum \tilde{c}_{i}\left(u-u_{0}\right)^{l}\|\phi\|_{W_{2,2}}^{2} .
\end{aligned}
$$

Let us choose the $c_{i}$ 's so that

$$
c_{1} \sup e^{4 \lambda} \leqq 1, \quad 2 c_{2} \sup e^{4 \lambda} \leqq c_{1}, \ldots, \quad l c_{l} \sup e^{4 \lambda} \leqq c_{l-1} .
$$

By Proposition 3.1 the constants $c_{\imath}$ can be estimated from above by a constant depending upon $\left\|\lambda\left(u_{0}\right)\right\|_{w_{2,2}}$. Equation (4.7), Proposition 3.1 and Proposition 
4.1 imply

$$
\frac{d E}{d u} \leqq C\left(1+\left(u-u_{0}\right)^{l}\right)\|\phi\|_{W_{2,2}}^{2}, \quad \phi=\lambda-\langle\lambda\rangle
$$

thus

$$
\begin{aligned}
E(u) & \leqq E\left(u_{0}\right)+C \int_{u_{0}}^{u}\left(1+\left(s-u_{0}\right)^{l}\right)\|\phi(s)\|_{W_{2,2}}^{2} d s \\
& =\left\|e^{2 \lambda}\left|\dot{\nabla}^{k} \lambda\right|\left(u_{0}\right)\right\|_{L_{2}}^{2}+C \int_{u_{0}}^{u}\left(1+\left(s-u_{0}\right)^{l}\right)\|\phi(s)\|_{W_{2,2}}^{2} d s,
\end{aligned}
$$

and the result follows.

Corollary 4.1. Let $\lambda$ satisfy (3.1). For every $m \geqq 0$ there exists a constant $C_{m}$ such that

$$
\forall u \geqq 1 \quad\|\lambda(u)\|_{W_{m, 2}} \leqq C_{m}
$$

Remark. $C_{m}$ depends upon $\left\|\lambda_{0}\right\|_{W_{2,2}}$.

Proof. It follows from Proposition 3.1 that (4.8) holds for $0 \leqq m \leqq 2$. Let $m>2$, thus $m=k+2 l$, with $k=1$ or $2, l \in \mathbb{N}$. Let $u \geqq 1$, from (4.6) with $u_{0}=u-1$ one finds

$$
\begin{aligned}
\left\|\dot{\nabla}^{m} \lambda(u)\right\|_{L_{2}}^{2} & \leqq C\left\{\left\|\left|\dot{\nabla}^{k} \lambda\right|(u-1)\right\|_{L_{2}}^{2}+\int_{u-1}^{u}\|(\lambda-\langle\lambda\rangle)(s)\|_{W_{2,2}}^{2} d s\right\} \\
& \leqq C^{\prime} . \square
\end{aligned}
$$

Corollary 4.2. Let $\lambda$ satisfy (3.1). For every $\Delta>0,0<\delta \leqq 1, m \in \mathbb{N}, u_{0} \geqq 0$, there exists a constant $C$ such that if

$$
\left\|\lambda\left(u_{0}\right)\right\|_{w_{2,2}} \leqq \delta
$$

then

$$
\left\|\lambda\left(u_{0}+\Delta\right)\right\|_{W_{m, 2}} \leqq C \delta
$$

Proof. Let

$$
F(u)=\int e^{-4 \lambda}\left|\dot{\nabla}^{2} \lambda\right|^{2} d \mu_{0} .
$$

From Proposition 3.1 and Lemma 4.1 it follows that

$$
\frac{d F(u)}{d u} \leqq C\|\phi(u)\|_{W_{2,2}}^{2} \text {. }
$$

From the inequality (cf. e.g. [1, Theorem 15.1', p. 703 and Proposition 3.2])

we have

$$
\|\phi\|_{W_{2,2}}^{2} \leqq C \int\left|\dot{\nabla}^{2} \lambda\right|^{2} d \mu_{0} \leqq C^{\prime} \int e^{-4 \lambda}\left|\dot{\nabla}^{2} \lambda\right|^{2} d \mu_{0}
$$

$$
\frac{d F(u)}{d u} \leqq C F(u)
$$


integrating in $u$ one obtains

$$
F(u) \leqq F\left(u_{0}\right) e^{C\left(u-u_{0}\right)},
$$

hence (4.11) and (4.10) lead to

Now

$$
u_{0} \leqq u \leqq u_{0}+\Delta, \quad\|\phi(u)\|_{W_{2,2}} \leqq C \delta .
$$

$$
\begin{aligned}
\left|e^{\langle 2 \lambda\rangle}-1\right| & =\frac{1}{A}\left|\int\left(e^{2\langle\lambda\rangle}-e^{2 \lambda}\right) d \mu_{0}\right| \\
& =\frac{e^{2\langle\lambda\rangle}}{A}\left|\int\left(1-e^{2 \phi}\right) d \mu_{0}\right| .
\end{aligned}
$$

Since $\langle\phi\rangle=0$ there exists $p \in{ }^{2} M$ such that $\phi(u, p)=0$. By Sobolev's embedding there exists $\alpha>0$ and a constant $C$ such that the $\alpha$-Hölder continuity modulus of $\phi$ is bounded by $C\|\phi\|_{W_{2,2}}$, thus

$$
\begin{aligned}
\left|1-e^{2 \phi}\right|(u, q) & =\left|\left(1-e^{2 \phi}\right)(u, q)-\left(1-e^{2 \phi}\right)(u, p)\right| \\
& \leqq C \sup _{q}|\phi(u, q)-\phi(u, p)| \\
& \leqq C^{\prime}\|\phi\|_{W_{2,2}},
\end{aligned}
$$

and (4.12) implies

$$
|\langle\lambda\rangle| \leqq C \delta
$$

which finally gives

$$
u_{0} \leqq u \leqq u_{0}+\Delta, \quad\|\lambda(u)\|_{W_{2,2}} \leqq C \delta,
$$

which establishes (4.9) for $m \leqq 2$. If $m>2$, let $m=k+2 l, k=1$ or $2, l \in \mathbb{N}$. Equation (4.6) with $u=u_{0}+\Delta$ and (4.13) imply

$$
\left\|\left|\dot{\nabla}^{m} \lambda\right|\left(u_{0}+\Delta\right)\right\|_{L_{2}}^{2} \leqq C \delta^{2}
$$

which gives (4.9).

\section{Convergence to a Constant Curvature Metric}

The results of the previous section show that all the $C^{k}$ norms of $\lambda, R(g)$, etc., are bounded for $u \geqq 1$. In this section we shall show that $R(g)$ asymptotically converges to a constant, exponentially fast with decay rate equal to the decay rate of the linearized equation ("modulo gauge").

Proposition 5.1. Let $\lambda$ satisfy (3.1) (with $T=\infty$ ), let $g_{i j}=e^{2 \lambda} \stackrel{\circ}{g}_{i j}$, where $\stackrel{\circ}{g}_{i j}$ is a constant curvature metric on ${ }^{2} M$, let $\mu$ be the smallest eigenvalue of $-\Delta_{0}$ satisfying $\mu>\max \left(0, R_{0}\right)$. The metric $g_{i j}$ converges exponentially fast to $\stackrel{g}{g}_{i j}$, up to a conformal diffeomorphism. More precisely, there exists a function $\lambda_{\infty} \in C^{\infty}\left({ }^{2} M\right)$ such that

$$
R\left(e^{2 \lambda \propto} \stackrel{\circ}{g}_{i j}\right)=R\left(\dot{g}_{i j}\right),
$$


and for all $k$ there exists a constant $C_{k}$ such that

$$
\begin{gathered}
\forall u \geqq 1, \quad\left\|\left(\lambda-\lambda_{\infty}\right)\right\|_{C^{k}\left({ }^{2} M\right)} \leqq C_{k} e^{-v u}, \\
v=\mu\left(\mu-R_{0}\right) .
\end{gathered}
$$

Remark. If ${ }^{2} M \neq S^{2}$, then $\lambda_{\infty}$ is a constant (necessarily equal to zero with our normalization conditions) and $\mu$ is the first non-zero eigenvalue of $-\Delta_{0}$. If ${ }^{2} M=S^{2}$, then $\lambda_{\infty}$ is in general not a constant, and $\mu$ is the second non-zero eigenvalue of $-\Delta_{0}$.

To prove Proposition 5.1 we shall use a series of auxiliary lemmata:

Lemma 5.1. Suppose that (5.1) holds with some constant $v$ satisfying $v>\frac{2}{3} \mu\left(\mu-R_{0}\right)$. Then (5.1) also holds with $v=\mu\left(\mu-R_{0}\right)$.

Proof. To avoid (non-essential) problems with non-linear subspaces of Banach spaces it is useful to replace $\lambda$ by

$$
h=e^{2 \lambda}-1 \text {. }
$$

It follows from (2.9) that

$$
\langle h\rangle=\frac{1}{A} \int h d \mu_{0}=0 .
$$

If ${ }^{2} M \neq S^{2}$ we shall write the equation for $h$ in the form

$$
\frac{\partial h}{\partial u}=L h+g(h)
$$

where $L$ is the linearization of $\frac{\partial h}{\partial u}$ at $h=0$,

and

$$
L \phi=-\Delta_{0}^{2} \phi-R_{0} \Delta_{0} \phi
$$

$$
g(h)=\Delta_{0} R+\Delta_{0}^{2} h+R_{0} \Delta_{0} h .
$$

In the case ${ }^{2} M=S^{2}$ we set

$$
\begin{gathered}
L \phi=-\Delta_{0}^{2} \phi-R_{0} \Delta_{0} \phi-\mu_{2}\left(\mu_{2}-R_{0}\right) \sum_{i} \phi_{i} \int_{2 M} \phi_{i} \phi d \mu_{0}, \\
g(h)=\Delta_{0} R+\Delta_{0}^{2} h+R_{0} \Delta_{0} h+X^{a}(h) \nabla_{a} h-R_{0} h \sum \alpha_{i}(h) \phi_{i}, \\
X^{a}(h)=\sum \alpha_{i}(h) \dot{g}^{a b} \nabla_{b} \phi_{i}, \\
\alpha_{i}(h)=\frac{1}{R_{0}} \mu_{2}\left(\mu_{2}-R_{0}\right) \int_{2 M} h \phi_{i} d \mu_{0},
\end{gathered}
$$

where the $\phi_{i}$ 's form an $L_{2}$-orthonormal basis of the first non-trivial eigenspace of $\Delta_{0}$,

$$
\Delta_{0} \phi_{i}=-R_{0} \phi_{i}=-\mu_{1} \phi_{i},
$$

and $\mu_{2}$ is the second non-trivial eigenvalue of $-\Delta_{0}$ (recall that on a two dimensional sphere with a constant curvature metric the eigenvalues of $-\Delta_{0}$ are of the 
form $\left.\mu_{l}=l(l+1) R_{0} / 2\right)$. As discussed in Appendix B, solutions of (5.4) on $S^{2}$ with $L, g$ defined in (5.7) are in one-to-one correspondence with solutions of the original Eq. (5.4) with $L, g$ defined by (5.5)-(5.6). Since $g(h)$ consists of terms which are at least quadratic in $h$, for all $k$ we have

$$
\|g(h)\|_{C^{k}\left({ }^{2} M\right)} \leqq C_{k} e^{-2 v u} .
$$

Equation (5.4) yields

$$
\frac{d}{d u} \int h^{2} d \mu_{0}=2 \int h L h d \mu_{0}+2 \int h g d \mu_{0} .
$$

Now

$$
\begin{aligned}
\int h L h d \mu_{0}= & -\int\left(\Delta_{0} h\right)^{2} d \mu_{0}+R_{0} \int(\dot{\nabla} h)^{2} d \mu_{0} \\
& -c \sum_{i}\left(\int \phi_{i} h d \mu_{0}\right)^{2}, \\
c= & \mu_{2}\left(\mu_{2}-R_{0}\right) \text { for } S^{2}, \quad c=0 \text { otherwise . }
\end{aligned}
$$

From (5.3) a straightforward eigenfunction expansion together with an approximation argument give

$$
\begin{gathered}
\int\left[\Delta_{0} h\right]^{2} d \mu_{0} \geqq \mu_{1}^{2} \int h^{2} d \mu_{0}, \\
\int(\stackrel{\nabla}{\nabla} h)^{2} d \mu_{0} \geqq \mu_{1} \int h^{2} d \mu_{0},
\end{gathered}
$$

which for $R_{0} \leqq 0(c=0)$ implies

$$
\begin{aligned}
\frac{d}{d u} \int h^{2} d \mu_{0} & \leqq-2 \mu_{1}\left(\mu_{1}-R_{0}\right) \int h^{2} d \mu_{0}+2 \int h g d \mu_{0} \\
& \leqq-2 \mu_{1}\left(\mu_{1}-R_{0}\right) \int h^{2} d \mu_{0}+C e^{-3 v u}
\end{aligned}
$$

In the case $c \neq 0$ a slightly more careful argument is needed. Let

$$
\begin{gathered}
h_{i}=\int_{{ }_{M}} \phi_{i} h d \mu_{0}, \\
P h=h-\sum_{i} h_{i} \phi_{i} .
\end{gathered}
$$

Let $\psi_{\alpha}$ denote an $L_{2}$ - orthonormal basis of eigenfunctions of $-\Delta_{0}$ with eigenvalues greater than or equal to $\mu_{2}$,

let

$$
-\Delta_{0} \psi_{\alpha}=\mu_{\alpha} \psi_{\alpha},
$$

$$
h_{\alpha}=\int_{2 M} \psi_{\alpha} h d \mu_{0} \text {. }
$$

We have, formally,

$$
\begin{aligned}
\int\left\{\left(\Delta_{0} h\right)^{2}-R_{0}\left(\nabla^{\circ} h\right)^{2}\right\} d \mu_{0} & =\sum \mu_{\alpha}\left(\mu_{\alpha}-R_{0}\right) h_{\alpha}^{2} \\
& \geqq \mu_{2}\left(\mu_{2}-R_{0}\right) \sum h_{\alpha}^{2} \\
& =\mu_{2}\left(\mu_{2}-R_{0}\right) \int(P h)^{2} d \mu_{0}
\end{aligned}
$$


(recall that $\mu_{1}=R_{0}$ ), and the above calculation can be easily justified by an approximation argument. Thus

$$
\begin{aligned}
\int_{S^{2}} h L h d \mu_{0} & \leqq-\mu_{2}\left(\mu_{2}-R_{0}\right) \int_{S^{2}}(P h)^{2} d \mu_{0}-\mu_{2}\left(\mu_{2}-R_{0}\right) \sum h_{i}^{2} \\
& =-\mu_{2}\left(\mu_{2}-R_{0}\right) \int_{S^{2}} h^{2} d \mu_{0} .
\end{aligned}
$$

Therefore in either case

$$
\frac{d}{d u} \int h^{2} d \mu_{0} \leqq-2 \mu\left(\mu-R_{0}\right) \int h^{2} d \mu_{0}+C e^{-3 v u}
$$

$\mu=\mu_{2}$ if ${ }^{2} M=S^{2}, \mu=\mu_{1}$ otherwise, so that

$$
\frac{d}{d u}\left(e^{2 \mu\left(\mu-R_{0}\right) u} \int h^{2} d \mu_{0}\right) \leqq C e^{\left[2 \mu\left(\mu-R_{0}\right)-3 v\right] u} .
$$

Integrating (5.10) in $u$ one has

$$
\left(\int h^{2} d \mu_{0}\right)(u) \leqq C e^{-2 \mu\left(\mu-R_{0}\right) u},
$$

because, by hypothesis,

$$
v>\frac{2 \mu\left(\mu-R_{0}\right)}{3}
$$

thus

$$
\|h\|_{L_{2}} \leqq C e^{-\mu\left(\mu-R_{0}\right) u}
$$

Note that

$$
\Delta_{0} L=L \Delta_{0},
$$

so that repeating the above argument for $\Delta_{0} h, \Delta_{0}^{2} h$, etc. (cf. (5.8)), one obtains

$$
\left\|\Delta_{0}^{k} h\right\|_{L_{2}} \leqq C_{k} e^{-\mu\left(\mu-R_{0}\right) u},
$$

which by elliptic estimates yields the claimed result.

Lemma 5.1 reduces the proof of (5.1)-(5.2) to a proof of (5.1) with some sufficiently large exponent ${ }^{6} v$. The following lemma shows that such an estimate holds if $\|h\|_{W_{2,2}}$ is sufficiently small:

Lemma 5.2. There exists $\varepsilon_{0}>0$ such that if for some $u_{0} \geqq 0$

$$
\left\|\lambda\left(u_{0}\right)\right\|_{w_{2,2}} \leqq \varepsilon_{0},
$$

then the hypotheses of Lemma 5.1 hold (and thus Proposition 5.1 holds).

Proof. Equation (5.11) and Corollary 4.2 imply that for any $k$ there exists a constant $C_{k}$ such that

$$
\left\|\lambda\left(u_{0}+1\right)\right\|_{w_{k, 2}} \leqq C_{k}\left\|\lambda\left(u_{0}\right)\right\|_{w_{2,2}},
$$

\footnotetext{
$\overline{{ }^{6}}$ Note that if $v$ in (5.1) is too small one can iterate a finite number of times the argument of the proof of Lemma 5.1 and again obtain the conclusion of Lemma 5.1; this will however not be needed in our proof
} 
if $\left\|\lambda\left(u_{0}\right)\right\|_{W_{2,2}}$ is small enough. Let $k=5$. If ${ }^{2} M \neq S^{2}$, it follows from e.g. a theorem by Lunardi [19] (cf. [23]) that for $\varepsilon_{0}$ small enough and for $u \geqq u_{0}+1$ we have

$$
\|\lambda(u)\|_{W_{2,2}} \leqq C(\beta) e^{-\beta u},
$$

with any $\beta<\mu\left(\mu-R_{0}\right)$, in particular (5.12) holds with $\beta=3 \mu\left(\mu-R_{0}\right) / 4$ $>2 \mu\left(\mu-R_{0}\right) / 3$. Corollary 4.2 implies now that for $u \geqq u_{0}+2$ and for all $k$

$$
\|\lambda(u)\|_{w_{k, 2}} \leqq C_{k}\|\lambda(u-1)\|_{w_{2,2}} \leqq C_{k}^{\prime} e^{-\beta u},
$$

which had to be established. If ${ }^{2} M=S^{2}$, a modification of the equation for $\lambda$ as in (5.7) (cf. Appendix B) allows one to apply Lunardi's theorem ${ }^{7}$ in the same way.

Proof of Proposition 5.1. We shall show that there always exists $u_{0}$ such that (5.11) holds. D. Singleton's Bondi mass-loss formula (A.12) implies

where

$$
m_{\infty}+12 \int_{0}^{\infty}\left(\int_{2 M} f^{-1}\left|D_{0} D_{0} f\right|^{2} d \mu_{0}\right)(u) d u=m_{0},
$$

$$
\begin{aligned}
& f \equiv e^{-\lambda} \\
& m_{0}=\int e^{3 \lambda_{0}} d \mu_{0}, \\
& m_{\infty}=\lim _{u \rightarrow \infty} \int e^{3 \lambda} d \mu_{0}
\end{aligned}
$$

[existence of $m_{\infty}$ follows from (A.12)], thus

$$
\int_{0}^{\infty} \int_{{ }_{2}} f^{-1}\left|D_{0} D_{0} f\right|^{2} d \mu_{0} d u<\infty,
$$

and since from Proposition 3.1 we have

$$
\left\|f^{-1}\right\|_{L_{\infty}} \leqq C, \quad\|f\|_{L_{\infty}} \leqq C,
$$

it follows that

$$
\int_{0}^{\infty} \int_{2 M}\left|D_{0} D_{0} f\right|^{2} d \mu_{0} d u<\infty .
$$

Equation (5.14) implies that there exists a sequence $u_{i} \rightarrow \infty$ such that

$$
\left(\int_{2_{M}}\left|D_{0} D_{0} f\right|^{2} d \mu_{0}\right)\left(u_{i}\right) \stackrel{i \rightarrow \infty}{\longrightarrow} 0 .
$$

From the identity (A.5) (with $D=D_{0}$ ) integrated over ${ }^{2} M$ and from (5.15) we have

$$
\left.\int\left[\left(\Delta_{0} f\right)^{2}-\mathrm{R}_{0}|\dot{\nabla} f|^{2}\right] d \mu_{0}\right|_{u=u_{i}} \stackrel{i \rightarrow \infty}{\longrightarrow} 0 .
$$

If $R_{0} \leqq 0(5.16)$ gives

$$
\left.\int\left(\Delta_{0} f\right)^{2} d \mu_{0}\right|_{u=u_{i}} \stackrel{i \rightarrow \infty}{\longrightarrow} 0
$$

\footnotetext{
7 In the $S^{2}$ case a similar argument has been independently used by D. Singleton [27] to prove global existence and convergence for small data
} 
and elliptic estimates together with (2.9) yield

$$
\left\|f\left(u_{i}\right)\right\|_{W_{2,2}} \stackrel{i \rightarrow \infty}{\longrightarrow} 0,
$$

which shows that there exists a $u_{0}$ such that (5.11) holds if ${ }^{2} M$ is not a sphere. When ${ }^{2} M=S^{2}$, a spectral expansion argument along the lines of (5.9) gives

$$
\int\left[\Delta_{0}(P f)\right]^{2} d \mu_{0} \leqq \frac{6}{5} \int\left[\left(\Delta_{0} f\right)^{2}-R_{0}|\nabla f|^{2}\right] d \mu_{0},
$$

thus (5.16) implies

$$
\left\|(P f)\left(u_{i}\right)\right\|_{W_{2,2}} \stackrel{i \rightarrow \infty}{\longrightarrow} 0 .
$$

The first spherical harmonics are restrictions of coordinate functions $x^{i}$ to $S^{2}$, when $S^{2}$ is embedded in $\mathbb{R}^{3}$ in a standard way, thus $f\left(u_{i}\right)$ can be written in the form

$$
f\left(u_{i}\right)=A_{i}+\mathbf{B}_{i} \mathbf{x}+(P f)\left(u_{i}\right),
$$

for some constants $A_{i}$ and some vectors $\mathbf{B}_{i}$. Equation (5.13) together with (5.17) imply

$$
\left|A_{i}\right|+\left|\mathbf{B}_{i}\right| \leqq C
$$

for $i$ large enough. It follows that we can choose a subsequence, still denoted $u_{i}$, such that

$$
A_{i} \rightarrow A_{\infty}, \quad \mathbf{B}_{i} \rightarrow \mathbf{B}_{\infty},
$$

and we have

$$
\left\|f\left(u_{i}\right)-A_{\infty}-\mathbf{B}_{\infty} \mathbf{x}\right\|_{W_{2,2}} \stackrel{i \rightarrow \infty}{\longrightarrow} 0
$$

The area conservation condition

$$
\int f^{-2} d \mu_{0}=\int 1 d \mu_{0}
$$

implies that there exists $\tau \in \mathbb{R}$ and a unit vector $\mathbf{n}$ such that

$$
f_{\infty} \equiv A_{\infty}+\mathbf{B}_{\infty} \mathbf{x}=\cosh \tau+\operatorname{sh} \tau \mathbf{n} \mathbf{x}
$$

which is precisely the condition for the existence of a conformal diffeomorphism $\phi$ of $S^{2}$ such that

$$
\phi^{*} \stackrel{\circ}{g}_{a b}=\left(f_{\infty}^{-2}\right) \stackrel{\circ}{g}_{a b}
$$

Consider the metric

let

$$
\bar{g}_{a b}(u)=\left(\phi^{-1}\right)^{*} g_{a b}(u),
$$

$$
\begin{aligned}
\bar{g}_{a b}(u) & =\bar{f}^{-2} \stackrel{\circ}{g}_{a b} \\
& =e^{2 \pi} \stackrel{\circ}{g}_{a b} .
\end{aligned}
$$

Equation (5.18) implies

$$
\left\|\bar{f}\left(u_{\imath}\right)-1\right\|_{W_{2,2}} \stackrel{i \rightarrow \infty}{\longrightarrow} 0,
$$


thus

$$
\left\|\bar{\lambda}\left(u_{i}\right)\right\|_{W_{2,2}} \stackrel{i \rightarrow \infty}{\longrightarrow} 0,
$$

and Proposition 5.1 follows by Lemma 5.2.

\section{A. Appendix}

In this Appendix we shall derive the inequalities (2.11) and (2.12) - these are key inequalities in all our results. From (2.7) and (2.8) by elementary manipulations one obtains

$$
\frac{d}{d u} \int_{{ }_{2} M} R^{2} d \mu_{g}=-2 \int\left\{\left(\Delta_{g} R\right)^{2}-R|\nabla R|^{2}\right\} d \mu_{g} .
$$

Following [18] we shall use the Kähler structure of ${ }^{2} M$ to show that the righthand side of (A.1) is nonpositive (cf. e.g. [13, 12] for an introduction to the theory of Kähler manifolds). Choose a holomorphic structure on ${ }^{2} M$, let $z=x+i y$; for any tensor field $t_{k \ldots l}^{i \ldots j}$ on ${ }^{2} M$, let

$$
\begin{gathered}
D t_{k \ldots l}^{i \ldots j} \equiv \nabla_{z} t_{k \ldots l}^{i \ldots j} \equiv \frac{1}{2}\left(\nabla_{x} t_{k \ldots l}^{i \ldots j}-i \nabla_{y} t_{k \ldots l}^{i \ldots j}\right) \\
\bar{D} t_{k \ldots l}^{i \ldots j} \equiv \nabla^{z} t_{k \ldots l}^{i \ldots J} \equiv g^{z \bar{z}} \nabla_{\bar{z}} t_{k \ldots l}^{i \ldots j} \equiv \frac{1}{2} g^{z \bar{z}}\left(\nabla_{x} t_{k \ldots l}^{i \ldots j}+i \nabla_{y} t_{k \ldots l}^{i \ldots j}\right) .
\end{gathered}
$$

For any smooth function $f$ on ${ }^{2} M$ we have

$$
\begin{gathered}
|\nabla f|^{2}=2 D f \bar{D} f, \\
\Delta f=2 D \bar{D} f=2 \bar{D} D f \\
\bar{D} D \bar{D} f-D \bar{D} \bar{D} f=-\frac{R}{2} \bar{D} f . \\
D D f \bar{D} \bar{D} f=\frac{1}{2}\left(\nabla^{i} \nabla^{j} f-\frac{1}{2} \Delta f g^{i j}\right)\left(\nabla_{i} \nabla_{j} f-\frac{1}{2} \Delta f g_{i j}\right) \geqq 0 .
\end{gathered}
$$

From (A.3)-(A.4) one finds the identity

$$
D \bar{D} f D \bar{D} f=\bar{D}(D f D \bar{D} f)-D(D f \bar{D} \bar{D} f)+D D f \bar{D} \bar{D} f+\frac{R}{2} D f \bar{D} f \text {. }
$$

Integrating (A.5) over ${ }^{2} M$ with $f=R$, the $D(\cdots)$ and $\bar{D}(\cdots)$ terms give no contribution (cf. e.g. [12]), and from (A.1)-(A.4), one finds

$$
\frac{d}{d u} \int R^{2} d \mu_{g}=-4 \int D D R \bar{D} \bar{D} R d \mu_{g} \leqq 0
$$

which together with (2.10) establishes (2.11). D. Singleton's inequality (2.12) requires some more work. From (2.5) and (2.6) one finds

$$
\begin{aligned}
\frac{d}{d u} \frac{1}{3} \int e^{3 \lambda} d \mu_{0}= & -\int e^{-\lambda}\left(\Delta_{0} \lambda\right)^{2} d \mu_{0} \\
& -\int e^{-\lambda} \Delta_{0} \lambda|\dot{\nabla} \lambda|^{2} d \mu_{0}+R_{0} \int e^{-\lambda}|\dot{\nabla} \lambda|^{2} d \mu_{0}
\end{aligned}
$$


$\left(R_{0}=\right.$ const $)$. Let

$$
f \equiv e^{-\lambda}
$$

(A.7) can be written as

$$
\begin{aligned}
\frac{d}{d u} \frac{1}{3} \int e^{3 \lambda} d \mu_{0} & =\int f^{-1}\left(\Delta_{0} f\right)^{2} d \mu_{0}+3 \int f^{-2} \Delta_{0} f|\dot{\nabla} f|^{2} d \mu_{0} \\
& +R_{0} \int f^{-1}|\dot{\nabla} f|^{2} d \mu_{0}-2 \int f^{-3}|\dot{\nabla} f|^{4} d \mu_{0} .
\end{aligned}
$$

Let $D_{0}, \bar{D}_{0}$ be the Kähler derivative operators of the metric $g_{0}$. One has the identities

$$
\begin{array}{r}
f^{-1} \bar{D}_{0} D_{0} f D_{0} \bar{D}_{0} f=\bar{D}_{0}\left(f^{-1} D_{0} f D_{0} \bar{D}_{0} f\right)-\mathrm{D}_{0}\left(f^{-1} D_{0} f \bar{D}_{0} \bar{D}_{0} f\right) \\
+f^{-1} D_{0} D_{0} f \bar{D}_{0} \bar{D}_{0} f+\frac{R_{0}}{2} f^{-1} D_{0} f \bar{D}_{0} f \\
+f^{-2} \bar{D}_{0} f D_{0} f D_{0} \bar{D}_{0} f-f^{-2} D_{0} f D_{0} f \bar{D}_{0} \bar{D}_{0} f, \\
f^{-2} D_{0} f D_{0} f \bar{D}_{0} \bar{D}_{0} f=\bar{D}_{0}\left(f^{-2} D_{0} f D_{0} f \bar{D}_{0} f\right) \\
+2 f^{-3} \bar{D}_{0} f D_{0} f \bar{D}_{0} f D_{0} f \\
-2 f^{-2} D_{0} f \bar{D}_{0} f \bar{D}_{0} D_{0} f .
\end{array}
$$

Integrating (A.9)-(A.10) over ${ }^{2} M$ leads to

$$
\begin{aligned}
\int f^{-1}\left(\Delta_{0} f\right)^{2} d \mu_{0}= & 4 \int f^{-1} D_{0} D_{0} f \bar{D}_{0} \bar{D}_{0} f d \mu_{0}+R_{0} \int f^{-1}|\dot{\nabla} f|^{2} d \mu_{0} \\
& +3 \int f^{-2}|\dot{\nabla} f|^{2} \Delta_{0} f d \mu_{0}-2 \int f^{-3}|\dot{\nabla} f|^{4} d \mu_{0} .
\end{aligned}
$$

Equation (A.11) inserted in (A.8) gives D. Singleton's formula [27],

$$
\frac{d}{d u} \int e^{3 \lambda} d \mu_{0}=-12 \int f^{-1} D_{0} D_{0} f \bar{D}_{0} \bar{D}_{0} f d \mu_{0} \leqq 0,
$$

which establishes (2.12). Let us mention that the integral

$$
\int e^{3 \lambda} d \mu_{0}
$$

is related to the Bondi mass of the metric (2.1) [27].

\section{B. Appendix}

When ${ }^{2} M=S^{2}$, the operator obtained by linearizing the right-hand side of the Robinson-Trautman equation has zero eigenvalues, which leads to difficulties when trying to prove convergence of the metric to a constant curvature metric. The origin of these difficulties may be traced back to the existence of non-trivial conformal motions of the sphere, as a result of which $\lambda$ in (2.3) is defined only up to the addition of the logarithm of a conformal factor for a conformal trans- 
formation of $S^{2}$. To handle this problem ${ }^{8}$, let $\phi(u)$ be a $u$-dependent conformal diffeomorphism of $\dot{g}_{a b}$, let

$$
\tilde{g}_{a b}(u)=\phi(u)^{*} g_{a b}(u) .
$$

We have

$$
\frac{\partial \tilde{g}_{a b}}{\partial u}=\phi(u)^{*}\left[\frac{\partial g_{a b}}{\partial u}+\mathscr{L}_{\phi_{*} X(u)} g_{a b}\right]=\phi(u)^{*} \frac{\partial g_{a b}}{\partial u}+\mathscr{L}_{X(u)} \tilde{g}_{a b},
$$

where

$$
X(u)=\left(\phi^{-1}\right)_{*} \frac{\partial \phi}{\partial u}
$$

is the $u$-dependent conformal Killing vector field generated by $\phi(u)$, and $\mathscr{L}_{X}$ denotes a Lie derivative. $X(u)$ is of the form

$$
X(u)=\sum_{i} \alpha_{i}(u) \dot{\nabla} \phi_{i},
$$

where the $\phi_{i}$ 's form an $L_{2}$-orthonormal basis of the first non-trivial eigenspace of $\Delta_{0}, \dot{\nabla}$ is the gradient operator of the metric $g$, and the $\alpha_{i}(u)$ 's are $u$-dependent constants. We have

$$
\mathscr{L}_{X} \stackrel{\circ}{g} a b=-R_{0} \sum \alpha_{i}(u) \phi_{i} \stackrel{\circ}{g}_{a b} .
$$

For a metric $g_{a b}(u)$ satisfying the Robinson-Trautman equation one obtains

$$
\frac{\partial \tilde{\lambda}}{\partial u}=\frac{1}{2}\left(\Delta_{g} R-R_{0} \sum \alpha_{i} \phi_{i}\right)+X^{a} \tilde{\lambda,},
$$

where $\tilde{\lambda}$ is defined by

$$
\tilde{g}_{a b}=e^{2 \tilde{\pi}} \dot{o}_{a b} .
$$

Let $F \in C^{\infty}(\mathbb{R})$ be any function such that $F(0)=0, F^{\prime}(0) \neq 0$. The choice

$$
\alpha_{i}(u)=\int_{2_{M}} F(\tilde{\lambda}(u, p)) \phi_{i}(p) d \mu_{0}
$$

is especially convenient because in this way one gets rid of zero eigenvalues of the operator which arises by linearizing the right-hand side of the modified Robinson-Trautman equation (B.3). Given $\tilde{\lambda}(u, p)$ satisfying (B.3), such that $\tilde{\lambda}(0, p)=\lambda_{0}(p)$, a solution of the original equation can be recovered by integrating $\phi(u)$ from (B.2), with $\phi(0)=i d, \alpha_{i}$ given by (B.4), and inverting (B.1) gives $g_{a b}(u)$. It is simple to check that if $\tilde{\lambda}$ converges exponentially in $C^{\infty}$ to 0 with some decay rate $\beta$, then $\phi$ will exponentially converge to a smooth conformal diffeomorphism $\phi_{\infty}$ of $S^{2}$ with decay rate $\beta$, and that $\lambda$ will exponentially converge to a limiting function $\lambda_{\infty}$ defined by

$$
e^{2 \lambda \infty} \stackrel{\circ}{g} a b_{a \infty}=\phi_{\infty}^{*} \stackrel{\circ}{g}_{a b}
$$

(cf. [27] for a more detailed exposition).

\footnotetext{
8 The same method as presented below has been independently used by Singleton [27] to get rid of the zero-eigenvalue problem
} 


\section{Appendix}

In this Appendix we shall prove some estimates use of which is made in Sect. 4. Let us define the convention, that for $i_{j} \in \mathbb{N}$ the "equality"

$$
f=g+“ \dot{\nabla}^{i_{1}} \lambda \cdots \dot{\nabla}^{i_{s}} \lambda ”
$$

is a shorthand for the statement that $f-g$ consists of a sum of terms of the form

$$
\text { const } \dot{\nabla}^{\alpha_{1}} \lambda \cdots \dot{\nabla}^{\alpha_{s}} \lambda, \quad\left|\alpha_{j}\right|=i_{j},
$$

in particular there exists a constant $C$ such that

$$
|f-g| \leqq C\left|\dot{\nabla}^{i_{1}} \lambda\right| \cdots\left|\dot{\nabla}^{i_{s}} \lambda\right|
$$

Lemma C.1. For any multi-index $\alpha=\left(a_{1}, \ldots, a_{l}\right), 0 \leqq|\alpha|=l$, we have

$$
\begin{aligned}
\frac{\partial \dot{\nabla}_{\alpha} \lambda}{\partial u} & =e^{-4 \lambda}\left(-\Delta_{0}^{2} \dot{\nabla}_{\alpha} \lambda+\stackrel{1}{\mathscr{R}}_{\alpha}+\stackrel{2}{\mathscr{R}}_{\alpha}+\stackrel{3}{\mathscr{R}}_{\alpha}\right), \\
\mathscr{R}_{\alpha} & =“ \dot{\nabla} \lambda \dot{\nabla}^{3+|\alpha|} \lambda " \\
\mathscr{\mathscr { R }}_{\alpha} & ="\left(1+\dot{\nabla} \lambda \dot{\nabla} \lambda+\dot{\nabla}^{2} \lambda\right) \dot{\nabla}^{2+|\alpha|} \lambda ", \\
\mathscr{R}_{\alpha} & =\sum_{\substack{s \geqq 2 \\
1 \leqq i_{j} \leqq 1+|\alpha| \\
i_{1}+\cdots+i_{s} \leqq|\alpha|+4}} \dot{\nabla}^{i_{1}} \lambda \ldots \dot{\nabla}^{i_{s}} \lambda .
\end{aligned}
$$

Proof. One shows by induction that

$$
\dot{\nabla}_{\alpha} \Delta_{0}^{2}=\Delta_{0}^{2} \dot{\nabla}_{\alpha}+\mathscr{R}_{\alpha},
$$

where $\Delta_{0}$ is the Laplacian acting on tensors, and $\mathscr{R}_{\alpha}$ is a sum of terms of the form

$$
R_{0} \dot{\nabla}_{\alpha_{1}} \cdots \dot{\nabla}_{\alpha_{s}} \lambda, \quad s=2+|\alpha|
$$

For $|\alpha|=0$ from (3.1) and (2.5) we have

$$
\begin{aligned}
\frac{\partial \lambda}{\partial u}= & \frac{1}{2} e^{-2 \lambda} \Delta_{0}\left\{e^{-2 \lambda}\left(R_{0}-2 \Delta_{0} \lambda\right)\right\} \\
= & e^{-4 \lambda}\left\{-\Delta_{0}^{2} \lambda+“ \dot{\nabla} \lambda \dot{\nabla}^{3} \lambda "+“\left(1+\dot{\nabla} \lambda \dot{\nabla} \lambda+\dot{\nabla}^{2} \lambda\right) \dot{\nabla}^{2} \lambda "\right. \\
& +“ \dot{\nabla} \lambda \dot{\nabla} \lambda "\} \\
\leqq & e^{-4 \lambda}\left\{-\Delta_{0}^{2} \lambda+\mathscr{\mathscr { R }}_{0}+\stackrel{\mathscr{R}}{\mathscr{R}}_{0}+\mathscr{\mathscr { R }}_{0}\right\}
\end{aligned}
$$

with $\stackrel{a}{\mathscr{R}}_{0}$ satisfying (C.2)-(C.4). An induction argument using (C.5) yields the result. 
Let us recall the well known Gagliardo-Nirenberg inequalities:

Proposition C.1 ([2], Theorem 3.70, p. 93). For $2 \leqq p \leqq \infty, 0 \leqq j<m$, there exists a constant $C$ such that the equality $\int_{2 M} f d \mu_{0}=0$ implies

$$
\left\|\dot{\nabla}^{j} f\right\|_{L_{p}} \leqq C\left\|\dot{\nabla}^{m} f\right\|_{L_{2}}^{a}\|f\|_{L_{2}}^{1-a}, \quad a=\frac{j+1-\frac{2}{p}}{m} .
$$

Proposition C.1 implies another well known result, which we prove here for completeness:

Lemma C.2. For $2 \leqq p<\infty, 2 \leqq k<l+2$, there exists a constant $C$ such that the equality $\int_{{ }_{2}} \phi d \mu_{0}=0$ implies

$$
\left\|\dot{\nabla}^{k} \phi\right\|_{L_{p}} \leqq C\left(\left\|\dot{\nabla}^{l+2} \phi\right\|_{L_{2}}^{a}\|\phi\|_{W_{2,2}}^{1-a}+\|\phi\|_{W_{2,2}}\right), \quad a=\frac{k-1-\frac{2}{p}}{l} .
$$

Proof. Let $f=\Delta_{0} \phi$, set $j=k-2$. By elliptic estimates ([1], Theorem 15.1', p. 703) we have

$$
\|\phi\|_{W_{k, p}} \leqq C\left(\|f\|_{W_{J, p}}+\|\phi\|_{L_{p}}\right) .
$$

Let us consider the case $k=2$ first. Equation (C.9), Proposition C.1, the Sobolev embedding and the definition of $f$ imply

$$
\begin{aligned}
\left\|\dot{\nabla}^{2} \phi\right\|_{L_{p}} & \leqq\|\phi\|_{W_{2, p}} \leqq C\left(\|f\|_{L_{p}}+\|\phi\|_{L_{p}}\right) \\
& \leqq C\left(\left\|\dot{\nabla}^{l} f\right\|_{L_{p}}^{a}\|f\|_{L_{p}}^{1-a}+\|\phi\|_{L_{p}}\right) \\
& \leqq C\left(\left\|\dot{\nabla}^{l+2} \phi\right\|_{L_{p}}^{a}\|\phi\|_{W_{2,2}}^{1-a}+\|\phi\|_{W_{2,2}}\right), \quad a=\frac{1-\frac{2}{p}}{l},
\end{aligned}
$$

which is (C.8) for $k=2$. For $k \geqq 3$ (C.9), Proposition C.1 and (C.10) similarly yield

$$
\begin{aligned}
\left\|\dot{\nabla}^{k} \phi\right\|_{L_{p}} & \leqq C\left(\|f\|_{W_{J, p}}+\|\phi\|_{L_{p}}\right) \leqq C\left(\left\|\dot{\nabla}^{j} f\right\|_{L_{p}}+\|f\|_{L_{p}}+\|\phi\|_{L_{p}}\right) \\
& \leqq C\left(\left\|\dot{\nabla}^{l} f\right\|_{L_{2}}^{a}\|f\|_{L_{2}}^{1-a}+\left\|\dot{\nabla}^{2} \phi\right\|_{L_{p}}+\|\phi\|_{L_{p}}\right) \\
& \leqq C\left(\left\|\dot{\nabla}^{l+2} \phi\right\|_{L_{2}}^{a}\|\phi\|_{W_{2,2}}^{1-a}+\left\|\dot{\nabla}^{l+2} \phi\right\|_{L_{2}}^{b}\|\phi\|_{W_{2,2}}^{1-b}+\|\phi\|_{W_{2,2}}\right),
\end{aligned}
$$

with

$$
a=\frac{k-1-\frac{2}{p}}{l}, \quad b=\frac{1-\frac{2}{p}}{l} .
$$

Yang's inequality

$$
x y \leqq \frac{x^{p}}{p}+\frac{y^{q}}{q}, \quad \frac{1}{p}+\frac{1}{q}=1
$$


with $x=\left\|\dot{\nabla}^{l+2} \phi\right\|_{L_{2}}^{b}\|\phi\|_{W_{2,2}}^{(1-a) b / a}, y=\|\phi\|_{W_{2,2}}^{(a-b) / a}, p=a / b, q=a /(a-b)$, allows one to get rid of the second term at the right-hand side of (C.11), and (C.8) follows.

Lemma C.3. Let

$$
E_{l}=\int_{2_{M}} e^{4 \lambda}\left|\dot{\nabla}^{l} \lambda\right|^{2} d \mu_{0} .
$$

For every $l \geqq 1, l \in \mathbb{N}$, we have

$$
\frac{d E_{l}}{d u} \leqq-2\left\|\dot{\nabla}^{l+2} \lambda\right\|_{L_{2}}^{2}+C\left(\left\|\dot{\nabla}^{l+1} \lambda\right\|_{L_{2}}^{2}+\stackrel{1}{\Phi_{l}}+\stackrel{2}{\Phi_{l}}\right),
$$

where

$$
\begin{gathered}
\stackrel{1}{\Phi}=\int_{2_{M}}|\dot{\nabla} \lambda|\left|\dot{\nabla}^{l+1} \lambda\right|\left|\dot{\nabla}^{l+2} \lambda\right| d \mu_{0}, \\
\Phi_{l}=\sum_{\substack{s \geqq 3 \\
1 \leqq i_{j} \leq l+1 \\
i_{1}+\cdots+i_{s} \leqq 2 l+4}} \int_{2_{M}}\left|\dot{\nabla}^{i_{1}} \lambda\right| \cdots\left|\dot{\nabla}^{i_{s}} \lambda\right| d \mu_{0} .
\end{gathered}
$$

Proof. From (C.1) we have

where

$$
\frac{d E_{l}}{d u}=I_{l}+\stackrel{0}{I} I_{l}+\stackrel{1}{I} I_{l}+\stackrel{2}{I} I_{l}+\stackrel{3}{I} I_{l}
$$

$$
\begin{aligned}
& I_{l}=4 \int_{{ }_{2}} e^{4 \lambda}\left|\dot{\nabla}^{l} \lambda\right| \frac{\partial \lambda}{\partial u} d \mu_{0}, \\
& \stackrel{0}{I I_{l}}=-2 \sum_{|\alpha|=l} \int_{2_{M}} \dot{\nabla}^{\alpha} \lambda \Delta_{0}^{2} \dot{\nabla}_{\alpha} \lambda d \mu_{0}, \\
& \stackrel{a}{I I_{l}}=2 \sum_{|\alpha|=l} \int_{2_{M}} \dot{\nabla}^{\alpha} \lambda \mathscr{R}_{\alpha}^{a} d \mu_{0}, \quad a=1, \ldots, 3 .
\end{aligned}
$$

The terms $I_{l}$ have to be estimated separately for $l=1,2$ and for $l \geqq 3$. If $l=1$, (C.6) and several integrations by part to get rid of the fourth and some of the third derivatives of $\lambda$ give

$$
\begin{aligned}
I_{1} & \leqq C\left\{\int_{2 M}|\dot{\nabla} \lambda|\left|\dot{\nabla}^{2} \lambda\right|\left|\dot{\nabla}^{3} \lambda\right| d \mu_{0}+\sum_{\substack{s \leqq 3 \\
1 \leqq i_{j} \leqq 2 \\
i_{1}+\cdots+i_{s} \leqq 6}} \int_{2_{M}}\left|\dot{\nabla}^{i_{1}} \lambda\right| \cdots\left|\dot{\nabla}^{i_{s}} \lambda\right| d \mu_{0}\right\} \\
& =C\left\{\stackrel{1}{\Phi}_{1}+\stackrel{2}{\Phi}_{1}\right\},
\end{aligned}
$$

$\stackrel{1}{\Phi_{1}}, \stackrel{2}{\Phi}_{1}$ given by (C.13)-(C.14). For $l=2$, an integration by parts to get rid of the fourth derivatives of $\lambda$ gives

$$
I_{2} \leqq C \stackrel{2}{\Phi_{2}}
$$


while for $l \geqq 3$ one directly obtains

$$
I_{l} \leqq C \stackrel{2}{\Phi_{l}}
$$

Consider now the terms $\stackrel{a}{I} I_{l}$. Commuting derivatives and integrating by parts $\stackrel{0}{I} I_{l}$ can be transformed to the form

$$
\begin{aligned}
\stackrel{0}{I} I_{l} & =-2 \sum_{|\alpha|=l+2} \int_{2 M} \dot{\nabla}^{\alpha} \lambda \dot{\nabla}_{\alpha} \lambda d \mu_{0}+\int_{{ }_{2}} \dot{\nabla}^{l} \lambda \dot{\nabla}^{l+2} \lambda d \mu_{0} " \\
& =-2\left\|\dot{\nabla}^{l+2} \lambda\right\|_{L_{2}}^{2}+\int_{{ }_{2}} \dot{\nabla}^{l+1} \lambda \dot{\nabla}^{l+1} \lambda d \mu_{0} ”,
\end{aligned}
$$

thus

$$
\stackrel{0}{I I} \leqq-2\left\|\dot{\nabla}^{l+2} \lambda\right\|_{L_{2}}^{2}+C\left\|\dot{\nabla}^{l+1} \lambda\right\|_{L_{2}}^{2}
$$

An integration by parts in $\stackrel{1}{I} I_{l}$ gives

$$
\begin{aligned}
\stackrel{1}{I}_{l} & =\text { “ } \int_{{ }_{2}} \dot{\nabla}^{l+3} \lambda \dot{\nabla}^{l} \lambda \dot{\nabla} \lambda d \mu_{0} " \\
& =\int_{2} \dot{\nabla}^{l+2} \lambda\left(\dot{\nabla}^{l+1} \lambda \dot{\nabla} \lambda+\dot{\nabla}^{l} \lambda \dot{\nabla}^{2} \lambda\right) d \mu_{0} " \\
& =\int_{{ }_{2}}\left\{\dot{\nabla}^{l+2} \lambda \dot{\nabla}^{l+1} \lambda \dot{\nabla} \lambda+\dot{\nabla}^{l+1} \lambda \dot{\nabla}^{l+1} \lambda \dot{\nabla}^{2} \lambda+\dot{\nabla}^{l+1} \lambda \dot{\nabla}^{l} \lambda \dot{\nabla}^{3} \lambda\right\} d \mu_{0} ",
\end{aligned}
$$

which yields

$$
\stackrel{1}{I} I_{l} \leqq C\left(\stackrel{1}{\Phi_{l}}+\stackrel{2}{\Phi_{l}}\right)
$$

Consider now $\stackrel{2}{I} I_{l}$ : an integration by parts to get rid of the $l+2^{\text {nd }}$ derivatives gives for $l \geqq 2$,

$$
\stackrel{2}{I} I_{l} \leqq C\left\{\left\|\dot{\nabla}^{l+1} \lambda\right\|_{L_{2}}^{2}+\stackrel{2}{\Phi_{l}}\right\}
$$

while for $l=1$ one has

$$
\stackrel{2}{I} I_{1} \leqq C\left\{\left\|\dot{\nabla}^{2} \lambda\right\|_{L_{2}}^{2}+\stackrel{1}{\Phi}{ }_{1}+\stackrel{2}{\Phi}\right\}
$$

Finally from the definition of $\stackrel{3}{\mathscr{R}} \alpha$ one obtains directly

$$
\stackrel{3}{I} I_{l} \leqq C \stackrel{2}{\Phi_{l}}
$$

and from what has been said (C.12)-(C.14) follow.

Lemma C.4. For every $l \in \mathbb{N} \geqq 1$, there exist a finite set $B$, a set of positive constants $\alpha_{\beta}, \gamma_{\beta}, \beta \in B$, and a constant $C$ such that

$$
\begin{gathered}
\frac{d E_{l}}{d u} \leqq-2\left\|\dot{\nabla}^{l+2} \lambda\right\|_{L_{2}}^{2}+C \sum_{\beta \in B}\left\|\dot{\nabla}^{l+2} \lambda\right\|_{L_{2}}^{\alpha_{\beta}}\|\phi\|_{W_{2,2}}^{\gamma_{\beta}}, \\
0<\alpha_{\beta}<2, \quad \gamma_{\beta} \geqq 2-\alpha_{\beta} .
\end{gathered}
$$


Proof. We have to estimate the three rightermost terms appearing in (C.12). Proposition C.1 yields immediately

$$
\left\|\dot{\nabla}^{l+1} \lambda\right\|_{L_{2}}^{2} \leqq C\left\|\dot{\nabla}^{l+2} \lambda\right\|_{L_{2}}^{2(l+1) /(l+2)}\|\phi\|_{L_{2}}^{2 /(l+2)},
$$

which is of the desired form. To estimate $\Phi_{l}$ we have

$$
\begin{aligned}
& \stackrel{1}{\Phi_{l}}=\int_{2_{M}}|\dot{\nabla} \lambda|\left|\dot{\nabla}^{l+1} \lambda\right|\left|\dot{\nabla}^{l+2} \lambda\right| d \mu_{0} \\
& \quad \leqq\|\dot{\nabla} \lambda\|_{L_{p_{1}}}\left\|\dot{\nabla}^{l+1} \lambda\right\|_{L_{p_{2}}}\left\|\dot{\nabla}^{l+2} \lambda\right\|_{L_{2}}, \quad \frac{1}{p_{1}}+\frac{1}{p_{2}}=\frac{1}{2},
\end{aligned}
$$

which by Sobolev embedding and Proposition C. 1 gives

$$
|\stackrel{1}{\Phi}| \leqq C\left\|\dot{\nabla}^{l+2} \lambda\right\|_{L_{2}}^{2-\frac{2}{p_{2}(l+2)}}\|\phi\|_{W_{2,2}}^{1+\frac{2}{p_{2}(l+2)}},
$$

which is of the form (C.15) and satisfies (C.16). To estimate $\stackrel{2}{\Phi_{l}}$ we shall use Lemma C.2. Let

$$
\begin{gathered}
\Psi \equiv \int_{2_{M}} \dot{\nabla}^{i_{1}} \lambda \cdots \dot{\nabla}^{i_{s}} \lambda d \mu_{0}, \\
s \geqq 3, \quad 1 \leqq i_{j} \leqq l+1, \quad i_{1}+\cdots+i_{s} \leqq 2 l+4,
\end{gathered}
$$

be one of the terms appearing in $\stackrel{2}{\Phi}$, let $k \geqq 0$ be the number of $i_{j}$ 's in (C.18) such that $i_{j}=1$. Reordering terms one may assume that for $1 \leqq j \leqq s-k$ we have $i_{j} \geqq 2$, thus

$$
\begin{gathered}
\Psi \leqq\|\dot{\nabla} \lambda\|_{L_{k p 0}}^{k}\left\|\dot{\nabla}^{i_{1}} \lambda\right\|_{L_{p 1}} \cdots\left\|\dot{\nabla}^{i_{s-k}} \lambda\right\|_{L_{p_{s-k}}}, \\
\frac{1}{p_{1}}+\cdots+\frac{1}{p_{s-k}}=1-\frac{1}{p_{0}}, \quad 2 \leqq i_{j} \leqq l+1, \quad i_{1}+\cdots+i_{s-k} \leqq 2 l+4-k .
\end{gathered}
$$

From (C.8) one obtains

$$
\begin{aligned}
\Psi & \leqq C\|\phi\|_{W_{2,2}}^{k} \prod_{j=1}^{s-k}\left(\left\|\dot{\nabla}^{l+2} \lambda\right\|_{L_{2}}^{a_{j}}\|\phi\|_{W_{2,2}}^{1-a_{j}}+\|\phi\|_{W_{2,2}}\right) \\
& =C \sum_{\beta \in B^{\prime}}\left\|\dot{\nabla}^{l+2} \lambda\right\|_{L_{2}}^{\alpha_{\beta}}\|\phi\|_{W_{2,2}}^{\gamma_{\beta}}, \quad a_{j}=\frac{i_{j}-1-\frac{2}{p_{j}}}{l},
\end{aligned}
$$

for some finite set $B^{\prime}$ of indices $\beta$. Note that for all $\beta \in B^{\prime}$ we have $\alpha_{\beta}+\gamma_{\beta} \geqq 3$ and that the largest exponent $\alpha_{\max }=\sup _{\beta \in B^{\prime}} \alpha_{\beta}$ in (C.19) is

$$
\alpha_{\max }=a_{1}+\cdots+a_{s-k} \leqq \frac{2 l+2-s+\frac{2}{p_{0}}}{l} .
$$

Choosing $p_{0}>2$ we have

$$
\alpha_{\max }<2,
$$

because $s \geqq 3$, and (C.15)-(C.16) follow. 
Acknowledgements. Useful discussions with B. Chow, F.H. Lin, and J. Shatah are acknowledged. The author is grateful to B. Schmidt and A. Rendall for comments and bibliographical advice. Special thanks are due to D. Singleton for presenting his results to the author prior to publication, and for pointing out an error in a previous version of this paper.

\section{References}

1. Agmon, S., Douglis, A., Nirenberg, L.: Commun. Pure Appl. Math. 12, 623 (1959)

2. Aubin, T.: Nonlinear analysis on manifolds, Monge-Ampère Equations. Berlin, Heidelberg, New York: Springer 1982

3. Calabi, E.: In: Seminar on differential geometry. Yau, S.T. (ed.). Princeton, NJ: Princeton University Press 1982

4. Chow, B.: J. Diff. Geom. (to appear)

5. Christodoulou, D.: Commun. Math. Phys. 105, 337 (1986); 106, 387 (1986); 109, 591 (1987); 109, 613 (1987)

6. Christodoulou, D., Klainermann, S.: Ann. Math. (to appear)

7. Chruściel, P.T.: Ann. Phys. (NY) 202, 100 (1990)

8. Chruściel, P.T., Isenberg, J., Moncrief, V.: Class. Quantum Grav. 7, 1671 (1990)

9. Cutler, C., Wald, R.M.: Class. Quantum Grav. 6, 453 (1989)

10. Foster, J., Newman, E.T.: J. Math. Phys. 8, 189 (1967)

11. Friedrich, H.: Commun. Math. Phys. 107, 587 (1987); Garching preprint MPA 402 (1988)

12. Futaki, A.: Kähler-Einstein metrics and integral invariants. Lecture Notes in Mathematics, Vol. 1314. Berlin, Heidelberg, New York: Springer 1988

13. Greene, R.: In: Differential geometry. Hansen, V.L. (ed.), Proceedings, Lyngby 1985, Lecture Notes in Mathematics, Vol. 1263. Berlin, Heidelberg, New York: Springer 1987

14. Hamilton, R.: In: Mathematics and general relativity. Isenberg, J. (ed.), Cont. Math., AMS 71, 237 (1988)

15. Hamilton, R.: Lectures at the Honolulu Conference on Heat Equations in Geometry, 1989

16. Isenberg, J., Moncrief, V.: Ann. Phys. (NY) 199, 84 (1990)

17. Lin, F.H.: Private communication

18. Lukács, B., Perjes, Z., Porter, J., Sebestyén, A.: Gen. Rel. Grav. 16, 691 (1984)

19. Lunardi, A.: Nonlin. Anal. 9, 563 (1985)

20. Moncrief, V.: Ann. Phys. 132, 87 (1981)

21. Osgood, B., Phillips, R., Sarnak, P.: J. Funct. Anal. 80, 148 (1988)

22. Perjes, Z.: Reported in [26]

23. Rendall, A.: Class. Quantum Grav. 5, 1339 (1988)

24. Robinson, I.: S.I.S.S.A. preprint 161, 1988

25. Robinson, I., Trautman, A.: Phys. Rev. Lett. 4, 431 (1960)

26. Schmidt, B.G.: Gen. Rel. Grav. 20, 65 (1988)

27. Singleton, D.: Ph. D. thesis; cf. also Class. Quantum Grav. 7, 1333 (1990)

28. Tod, K.P.: Class. Quantum Grav. 8, 1159 (1989)

29. Vandyck, M.A.J.: Class. Quantum Grav. 4, 759 (1987)

Communicated by S.-T. Yau 
